\title{
Responsabilidad social en estudiantes de medicina: experiencia de una sociedad científica estudiantil peruana
}

\author{
Social responsibility of students of medicine: experience in a scientific society of
}

Peruvian students

\author{
Joel Chalco-Huamán ${ }^{1,2, a}$, Juliana Zavala-Portugal ${ }^{1,2, a}$, Cristian Andonaire-Munaico ${ }^{1,3, b}$ \\ Facultad de Medicina, Universidad Nacional Mayor de San Marcos, Lima, Perú. \\ ${ }^{2}$ Sociedad Cientifica de San Fernando, Facultad de Medicina, Universidad Nacional Mayor de San Marcos, Lima, Perú. \\ ${ }^{3}$ Servicio de Pediatría, Hospital III Emergencias Pediátricas, Ministerio de Salud, Lima, Perú. \\ ${ }^{a}$ Estudiante de medicina; ${ }^{b}$ Médico, especialista en pediatria.
}

An Fac med. 2016;77(1):69 / http://dx.doi.org/10.15381/anales.v77i1.11559

\section{SR. EDITOR:}

La responsabilidad social ha intentado instaurarse como base de la estrategia educativa en las facultades de medicina a nivel mundial, puesto que, como formadoras de potenciales profesionales de salud, son actores clave en el desarrollo de una fuerza laboral sanitaria consciente de su realidad. Sin embargo, existen facultades que dejan de lado la importancia de formar agentes que generen un verdadero impacto en su sociedad y solo se interesan por sus necesidades educativas o comerciales ${ }^{(1)}$.

Países como Canadá y EE UU han tomado conciencia de la importancia de la responsabilidad social implementando nuevas estrategias curriculares en sus facultades ${ }^{(2)}$. En tanto, en América Latina, la disparidad en salud, así como el desinterés de médicos para trabajar en comunidades rurales, llama a incentivar la responsabilidad social. Pocos países han valorado el impacto positivo de esta en el fortalecimiento de su salud pública, en los que además cobra relevancia frente a la acelerada creación de facultades de medicina en toda la región y el gran impacto que se lograría a partir de ellas ${ }^{(3)}$.

Incentivados por las disparidades en salud, varias asociaciones estudiantiles afiliadas a la Federación Latinoamérica de Sociedades Científicas de Estudiantes de Medicina (FELSO. CEM) promueven la responsabilidad social, por medio de iniciativas como el
Campamento Universitario Multidisciplinario de Investigación y Servicio (CUMIS) ${ }^{(4)}$, que sobre todo los acerca a una realidad sociocultural distinta, sensibilizándolos y ampliándoles el panorama sobre la realidad de sus sistemas de salud. La Sociedad Científica de San Fernando (SCSF), afiliada a FELSOCEM, organizó en 1999 el primer CUMIS en Perú y hasta la fecha ha realizado 27 intervenciones, liderando la realización de este tipo de actividades a nivel nacional.

El CUMIS de SCSF se presenta como una oportunidad para incentivar la responsabilidad social en los estudiantes del área de salud, quienes participan en la planificación y ejecución de intervenciones de prevención y promoción de la salud en las comunidades visitadas. Asimismo, los docentes de la facultad tienen un rol importante al capacitar a los estudiantes, bajo un enfoque de atención primaria en salud, sobre distintos temas de énfasis como la promoción de vivienda saludable y planificación familiar, y la prevención de enfermedad diarreica y respiratoria aguda, desnutrición, anemia, salud bucal y mental. De este modo, por medio de charlas y dinámicas, los estudiantes buscan educar y concientizar a las comunidades visitadas.

Estas iniciativas estudiantiles son oportunidades que deben ser fomentadas por las facultades de medicina en América Latina, ya que además de generar un impacto en las comunidades visitadas permite a los estudiantes ser formados para el servicio y ejercicio en su propia realidad y, con ello, alcanzar una formación profesional integral.

\section{REFERENCIAS BIBLIOGRÁFICAS}

1. Boelen C, Woollard RF. Global consensus for social accountability of medical schools. Educ Med. 2011;14(1):7-14. Disponible en:

2. http://healthsocialaccountability.sites.olt.ubc.ca/ files/2011/06/11-06-07-GCSA-English-pdf-style.pdf

3. Murray RB, Larkins S, Russell H, Ewen S, Prideaux D. Medical schools as agents of change: socially accountable medical education. Med J Aust. 2012 Jun 4;196(10):653. Disponible en:

4. https://www.mja.com.au/journal/2012/196/10/medical-schools-agents-change-socially-accountablemedical-education.

5. Puschel K, Rojas P, Erazo A, Thompson B, Lopez J, Barros J. Social accountability of medical schools and academic primary care training in Latin America: principles but not practice. Fam Pract. 2014 Aug;31(4):399-408. doi: 10.1093/fampra/cmu010. Disponible en: http://fampra.oxfordjournals.org/ content/31/4/399.long.

6. Escalante L, Cueva L, Linares E, Blossiers C. Experiencias de trabajo en actividades en atención primaria de salud realizadas por estudiantes de medicina de Perú. CIMEL.2010;15(1):14-8. Disponible en:

7. http://revistas.concytec.gob.pe/scielo.php?pid= S168083982010000100004\&script=sci_arttext.

Carta recibida el 23 de septiembre de 2015.

Fuentes de financiamiento: autofinanciado.

Conflictos de interés: los autores declaran no tener conflictos de interés.

Correspondencia:

Joel Chalco-Huamán

Dirección: Pasaje Odriozola 176, Dpto. 402, La

Victoria, Lima, Perú

Teléfono: +511956035912

Correo electrónico: chalco.joel@gmail.com 